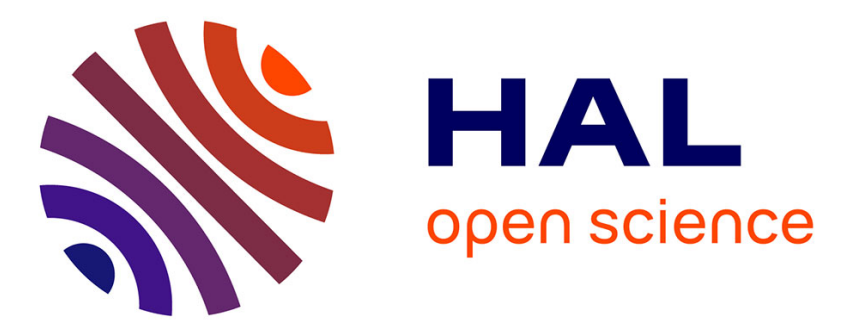

\title{
Contrôle des activités illégales en présence d'un biais d'optimisme
}

Nicolas Jacquemet, Jean-Louis Rullière, Isabelle Vialle

\section{To cite this version:}

Nicolas Jacquemet, Jean-Louis Rullière, Isabelle Vialle. Contrôle des activités illégales en présence d'un biais d'optimisme. Revue Economique, 2007, 58 (3), pp.555-564. 10.3917/reco.583.0555 . halshs-00259460

\section{HAL Id: halshs-00259460 \\ https://shs.hal.science/halshs-00259460}

Submitted on 7 Apr 2009

HAL is a multi-disciplinary open access archive for the deposit and dissemination of scientific research documents, whether they are published or not. The documents may come from teaching and research institutions in France or abroad, or from public or private research centers.
L'archive ouverte pluridisciplinaire HAL, est destinée au dépôt et à la diffusion de documents scientifiques de niveau recherche, publiés ou non, émanant des établissements d'enseignement et de recherche français ou étrangers, des laboratoires publics ou privés. 


\title{
Contrôle des activités illégales en présence d'un biais d'optimisme
}

\author{
Nicolas Jacquemet * Jean-Louis Rullière ** Isabelle Vialle **
}

Le contrôle est l'un des instruments majeur de répression des activités illégales. Cette dimension est intégrée aux analyses économiques du crime sous la forme d'une probabilité de détection et fondée seulement sur l'aversion au risque. La psychologie cognitive montre que la perception des probabilités peut elle-même varier d'un individu à l'autre. Cet article propose une analyse expérimentale de ce type d'erreur de perception, qualifiée de biais d'optimisme, à partir d'une série de choix entre une activité légale et une activité illégale. Les traitements expérimentaux évaluent la mesure dans laquelle les modalités de contrôle peuvent, à probabilité donnée, influencer les biais d'optimisme. Les résultats suggèrent que les politiques de communication de lutte contre les activités frauduleuses doivent favoriser la perception idiosyncrasique du risque.

\section{MONITORING ILLEGAL ACTIVITIES WITH OPTIMISTIC BIAS}

Although expensive, monitoring is the most efficient mean to repress a criminal activity. This is typically included in economic models of crime thanks to a probability of detection, and only based on risk aversion. According to recent results in psychology literature, the perception of this probability can however be specific to each individual. This paper experimentally investigates the existence of such errors in probabilities forecasts, often labelled "optimism bias". The experiment relies on choices between a no risky (legal) activity and an illegal activity -- randomly penalized by a fine. The experimental treatments assess whether the way the monitoring policy is announced can affect the optimism bias. The results provide a guide into costless devices to undermine illegal activities, grounded on an idiosyncratic perception of risk.

Classification JEL : C91, C92, D82, H21, H26

\section{INTRODUCTION}

Depuis les travaux de Becker (1968), l'analyse économique des activités illégales repose sur une comparaison entre les niveaux d'utilité respectifs de l'honnêteté et de la fraude. Dans ce cadre, les variables pertinentes pour l'analyse sont les gains et pertes associés à chacune des activités (légale et illégale), dans lesquelles interviennent notamment les éléments de la politique de détection menée par l'agence publique de lutte contre les activités illégales: le niveau des sanctions ainsi que la probabilité de détection des comportements frauduleux. C'est, en particulier, en raison de cette dernière dimension que le calcul qui fonde les décisions d'illégalité s'appuie sur des fonctions d'utilité en avenir incertain. Le degré d'aversion au risque des individus apparaît alors comme l'explication centrale de l'hétérogénéité observée dans les décisions d’illégalité: la fraude existe sans pour autant qu'elle soit généralisée ${ }^{1}$.

\footnotetext{
* Panthéon - Sorbonne Economie (CNRS - Université Paris 1) et Ecole d’Economie de Paris. Maison des Sciences Economiques, Bureau 420, 106 Bd. de l'hôpital, 75647 Paris Cedex 13, France. nicolas.jacquemet@univ-paris1.fr.

** GATE (CNRS - Université de Lyon). 93, chemin des mouilles, 69131 Ecully Cedex, France. Emails : rulliere@gate.cnrs.fr ; vialle@gate.cnrs.fr.

Nous tenons à remercier Alain Gubian, Marianne Pauchet, Nadia Joubert et Bernard Tapie pour leurs remarques avisées ainsi que le soutien financier de l’Agence Centrale des Organismes de Sécurité Sociale (ACOSS). Nos remerciements vont aussi à Nathan Berg, Gerd Gigerenzer, Erik Hoelzl et Philippe Delquié ; ainsi que les participants des sessions de l'ESA 2006 à Nottingham et du Congrès de l'AFSE pour leurs remarques et conseils. Nos remerciements vont aussi à Romain Zeiliger pour la programmation informatique du protocole. Bien évidemment, nous restons seuls responsables d'éventuelles erreurs ou omissions. Enfin, il est possible de se procurer sur simple demande le protocole ainsi que les données complètes des expérimentations.

${ }^{1}$ Par exemple le programme américain Taxpayer Compliance Measurement Program. (Internal Revenue Service, 1996) a estimé en 1988 sur 50000 ménages américains qu'environ 40\% des foyers américains procèdent intentionnellement à une sous-déclaration de leurs impôts, 53\% se conforment normalement à la loi et 7\% sur-contribuent en dépassant leur montant d'imposition.
} 
Du côté de l'agence de contrôle, la politique de lutte contre la fraude s'adosse donc sur l'aversion au risque de la population contrôlée. Par ce mécanisme, on voit donc tout l'intérêt de définir la politique de contrôle sur une variable psychologique individuelle. Dès lors, la question qui s'impose consiste à savoir s'il n'existe pas d'autres variables psychologiques qui pourraient elles aussi jouer un rôle et ainsi être aussi susceptibles de renforcer l'efficacité de la politique de contrôle.

Comme le suggèrent un certain nombre de travaux récents à la frontière de l'économie et de la psychologie, le contenu informationnel des probabilités associées à une loterie peut notamment être, en lui-même, extrêmement variable d'un individu à un autre. Un biais d'optimisme, en particulier, tend à déformer les probabilités à partir desquelles les décisions sont prises. Cette contribution s'intéresse au parti que peut tirer l'efficacité du contrôle de ces biais de perception. Il s'agit plus précisément d'évaluer la mesure dans laquelle la politique de communication influence l'optimisme (déformation des probabilités) d'individus confrontés à un choix d'illégalité. Il est important de noter ici que le choix de la nature des informations à diffuser est en général peu, voire non coûteux, alors que les effets économiques de ces choix sur les comportements à risque (comme celui du fraudeur) peuvent s’avérer très onéreux pour la collectivité.

Sur le plan méthodologique, la discrétion avec laquelle les activités illégales sont en général exercées rend complexe leur analyse empirique. Seules sont observables, en particulier, les activités illégales découvertes, i.e. celles qui ont échoué. Par ailleurs, en l'absence de changement exogène dans l'environnement -- réforme par exemple -- les données réelles ne permettent pas d'observer les ajustements marginaux du comportement aux variations de l'environnement. Nous optons par conséquent pour un traitement expérimental, qui permet de s'affranchir de ces deux contraintes.

Le protocole expérimental repose sur une succession de décisions indépendantes en environnement risqué. Deux traitements sont proposés, qui se distinguent par les modalités d'annonce des paramètres du contrôle, sous la forme d'une valeur (une probabilité) ou d'un nombre (nombre d'individus désignés aléatoirement et contrôlés). Toutes choses égales par ailleurs, les décisions de fraude apparaissent fortement sensibles à ces modalités d'annonce du contrôle.

La prochaine section propose un aperçu des apports de la psychologie cognitive à l'analyse économique du risque, qui ont permis d'apprécier l'impact économique des biais de perception. La section qui suit présente le protocole expérimental, destiné à mettre en évidence l'effet de la politique de communication sur les décisions de fraude. Les comportements observés dans le cadre des expériences en confirment l'influence. L'article se conclut enfin sur des éléments d'inflexion des politiques de répression de la fraude.

\section{APPORT DE LA PSYCHOLOGIE A L'ANALYSE ECONOMIQUE DE L'OPTIMISME}

Les psychologues ont depuis longtemps souligné le fait que les hommes maintiennent une image d'euxmêmes et de leurs perspectives futures qui n’est pas toujours fidèle à la réalité. La perception qu'un être humain a de lui-même est fréquemment sujette à des biais potentiels comme la sur-confiance. Une de ses manifestations possibles est plus connue sous le nom de l'effet meilleur que la moyenne (Better-Than-Average Effect) : dans un groupe, si plus de la moitié des individus se déclarent meilleurs ou avoir plus de chance de connaître un événement désirable que le membre médian, le groupe est globalement sur-confiant. Les erreurs de calibrage (miscalibration) sont une autre forme de sur-confiance par laquelle les individus surestiment la précision de leurs évaluations. La sur-confiance peut également se manifester par une illusion du contrôle : les individus tendent à surestimer le degré avec lequel ils influencent un résultat ou un évènement. Enfin, la sur-confiance peut se traduire par des biais dans l'attribution causale : les individus attribuent leurs succès à eux-mêmes et leurs échecs à la malchance. En fonction des variables qu'elles affectent, toutes ces formes de sur-confiance sont qualifiées d'image positive de soi ou d'optimisme. A l'inverse, on parle de sous-confiance et donc d'image négative de soi ou de pessimisme.

L'image positive de soi recouvre les erreurs de perception sur une caractéristique individuelle comme le niveau de compétences. Elle peut être révélée par l'effet meilleur que la moyenne en administrant des questionnaires d'auto-évaluation. Par exemple, les travaux d'Alicke (1985) révèlent que la majorité des individus surestime des caractéristiques individuelles, telles que l'habileté ou la ponctualité. Les psychologues ont aussi démontré que les gens maintiennent une image positive d'eux-mêmes à travers les erreurs de calibrage : dans une expérience, Fischhoff, Slovic et Lichtenstein (1977) demandent aux sujets d'indiquer la probabilité que leurs réponses à une série de questions soient correctes. Cette étude révèle que les participants surévaluent fortement la précision de leurs réponses. 
L'optimisme désigne quant à lui les biais qui se traduisent par une perception erronée d'événements futurs, typiquement des probabilités d'occurrence. Concrètement, un individu optimiste pense qu'il a plus (moins) de chance de connaître des évènements favorables (défavorables) que les autres. Comme pour l'image positive de soi, les psychologues ont étudié les biais d'optimisme à l'aide d'auto-évaluations qui concluent à l'effet meilleur que la moyenne. L'expérience originelle de Weinstein (1980) révèle que les individus surestiment la probabilité d'évènements positifs, tels qu'être propriétaire d'une maison ou avoir un enfant surdoué, alors qu'ils sousestiment la probabilité des évènements négatifs, tels qu'avoir un cancer ou être victime d'un cambriolage. Ces expérimentations concluent également que le biais est d'autant plus important que l'événement est perçu comme contrôlable; le biais persiste même pour des évènements exogènes et qui sont perçus comme tel par les individus.

Ces biais ne sont pas sans effet sur les comportements des agents. Par conséquent, ces phénomènes d'ordre psychologique ont été introduits dans les recherches en économie, depuis la fin des années 90 . Les modèles développés en finance ont été les premiers à introduire la sur-confiance pour expliquer les anomalies de marché. Dans ces travaux, la sur-confiance prend la forme d'un mauvais calibrage, au sens où les traders surestiment la précision de leur signal privé. Gervais et Odean (2001) développent un modèle dynamique dans lequel la surconfiance émerge de manière endogène, du fait que les traders surpondèrent le poids des signaux informatifs lorsqu'ils révisent leurs croyances sur leur habileté ; ce qui entraîne un accroissement de la volatilité des prix. Les biais de sur-confiance ont également été intégrés à d'autres domaines de l'économie, en particulier l'économie des ressources humaines, où son impact est examiné, par exemple, sur le choix de l'effort à l'origine de la performance (Bénabou et Tirole, 2002) ou sur la différenciation salariale (Fang et Moscarini, 2005).

Ces contributions théoriques prouvent tout le bénéfice qui peut être tiré de l'intégration des résultats issus de la psychologie. Les expériences menées en psychologie, présentées plus haut, sont cependant soumises à des critiques dont la plus importante s'inscrit dans un débat entre expérimentalistes en économie et en psychologie : les résultats sont obtenus sans introduire d'incitant monétaire, et le comportement observé n'est donc imputable qu'à la motivation intrinsèque des participants, qui reste difficile à apprécier. Par ailleurs, les questionnaires sont en général contextualisés ; il est par conséquent difficile d’en tirer des enseignements généraux, dans la mesure où il n'est pas possible d'isoler l'effet de contexte.

Les expériences réalisées en économie permettent de répondre à ces critiques. D’un point de vue historique, il convient cependant de signaler les résultats expérimentaux provenant des analystes financiers. La sur-réaction ou la sous-réaction sur un marché financier expérimental dépend de la fiabilité de l'information des investisseurs. Ces travaux montrent alors qu'un investisseur qui a une information imparfaite sur la fiabilité de son information va surestimer la crédibilité d'une information fortement invraisemblable et va sous-estimer la crédibilité d'une information fortement vraisemblable. (Griffin et Tversky, 1992). Dans le même sens, Biais, Hilton, Mazurier et Pouget (2005) mesurent le degré d'excès de confiance de 245 participants placés sur un marché financier expérimental avec asymétrie d'information, permettant d'apprécier les erreurs de calibrage. Les résultats indiquent que les sujets sous-estiment l'incertitude conditionnelle des actifs financiers en raison du biais de calibrage, et se rendent ainsi plus vulnérables à la malédiction du vainqueur.

Les économistes ont aussi répliqué l'effet meilleur que la moyenne issu de la littérature en psychologie. Initialement, Camerer et Lovallo (1999) analysent les surcapacités productives sur un marché en termes de biais de sur confiance de la part des entrants quant à la profitabilité du marché. Plus récemment, Hoelzl et Rustichini (2005) proposent une expérience qui a le mérite d'offrir une méthodologie originale dont nous avons pu nous inspirer en partie pour appréhender une mesure du biais d'optimisme dans les décisions de fraude. Leur contribution fournit un test direct de la sur-confiance relative à partir d'une procédure de vote sur le mode de rémunération monétaire des participants. Entre une période d'apprentissage d'une épreuve d'effort individuel (effort réel, sous forme de test de vocabulaire) et la participation effective à cette épreuve, deux modes de rémunération alternatifs pour l'effort sont soumis au vote :

1. Paiement aléatoire: la rémunération s'effectue selon un lancé de dés qui attribue une rémunération fixe (150 jetons) à la moitié des participants.

2. Paiement à la performance : les participants dont le niveau d'effort dépasse la valeur médiane du groupe reçoivent une rémunération fixe (150 jetons).

Les auteurs constatent que plus de la moitié des participants sélectionnent le paiement à la performance, révélant la sur-confiance globale des participants puisque plus de la moitié d'entre eux évalue leur performance au dessus de la valeur médiane.

Bien que la présence d'optimisme semble être attestée par des résultats en psychologie, peu de travaux ont à ce jour tiré les conséquences de ce type de biais de perception sur les décisions de se livrer à des activités illégales. C'est dans ce cadre que s'inscrit l'expérience proposée ici. 


\section{PROTOCOLE DE L’EXPERIENCE}

Afin de mettre en évidence l'influence de la politique de communication sur les décisions de fraude, l'expérience est constituée de deux parties, entre lesquelles seule cette dimension est modifiée. Au sein de chaque partie, les participants sont confrontés à différents choix de loterie. L'aversion au risque est donc une dimension importante de la prise de décision. Une étape préliminaire de mesure de l'aversion au risque est donc ajoutée à ces deux parties ; et elle consiste à répliquer le protocole standard de Holt et Laury (2005). Une mesure d'aversion au risque est déduite pour chaque individu à partir de dix choix séquentiels entre deux loteries.

Chacune de ces 2 parties comprend 22 périodes. Au début de chaque période, chaque participant reçoit 20 jetons qui doivent être alloués entre deux options. La première (option A) procure un gain de $15 \mathrm{ECU}^{2}$-- qui correspond à une activité légale ; la deuxième (option $\mathrm{B}$ ) est une loterie aléatoire : l'illégalité procure un gain élevé, de $20 \mathrm{ECU}$; en cas de détection, le gain est ramené à 15 ECU et soumis à une amende d'un montant $F$. Le déroulement des périodes est le même dans les deux parties. Les modalités du contrôle (i.e. la probabilité d’amende dans la loterie B), en revanche, sont différentes :

- Dans le traitement «Urne individuelle », un tirage au sort est effectué à la fin de la période pour chaque participant selon la probabilité $p$.

- Dans le traitement « Désignation », $n$ participants parmi les 20 présents sont sélectionnés aléatoirement.

Les 22 périodes conduites dans chaque partie correspondent à toutes les combinaisons possibles entre deux niveaux d'amende $(\mathrm{F}=10$ ou 14) et 11 niveaux de probabilité de contrôle ( $p=n / 20=5 \%, 10 \%, 15 \%, 20 \%$, 25\%, 30\%, 35\%, 40\%, 45\%, 50\% ou 55\%). La séquence des périodes est aléatoire afin de contrôler les effets d'ordre. Toutes ces informations (modalités du contrôle au début de chaque partie, $p$ et $F$ au début de chaque période) sont annoncées publiquement aux participants. Pour éviter les effets de feedback et d'apprentissage qui en découlent au cours de la session, les scores réalisés à chaque période ainsi que les résultats du contrôle ne sont révélés qu’à la fin de la session expérimentale sous la forme d'un tableau récapitulatif.

Les sessions expérimentales ont été conduites en juin 2006 au sein du laboratoire GATE (Groupe d'Analyse et de Théorie Economique), à Lyon ${ }^{3}$. Ces sessions ont réuni un total de 100 participants (5 sessions comprenant 20 participants chacune), constitués principalement d'étudiants inscrits à l'ITECH (Institut Textile et Chimique de Lyon), à l'EM (Ecole de Management de Lyon), à l'Ecole Centrale de Lyon. Les instructions sont distribuées et lues publiquement au début de chaque partie. Un questionnaire pré-expérimental est en outre distribué aux sujets afin de vérifier que les règles du jeu sont correctement comprises. La session s'achève par un questionnaire post-expérimental permettant de recueillir les caractéristiques individuelles des sujets. Une session dure en moyenne 90 minutes, incluant la lecture des instructions, les questionnaires et le paiement des sujets.

Afin d'éliminer les effets de richesse, les paiements sont basés sur l'une seulement des décisions prises au cours de l'expérience. Cette décision est sélectionnée par tirage au sort, pour chaque participant, à la fin de la session. Ce gain en ECU est converti selon le taux de 30 ECU pour $1 €$. A ce gain s'ajoute un forfait de participation de $7 €$. Les gains sont versés de façon privée, à la fin de l'expérience. La moyenne des gains par participant s'élève à $16 €$.

\section{OPTIMISME ET MODALITES DU CONTROLE}

Les variations de comportement entre les deux traitements Urne individuelle et Désignation peuvent sans ambiguïté être interprétées comme une déviation par rapport à l’hypothèse traditionnelle de rationalité. Un participant rationnel doit en effet affecter le même nombre de jetons à l'activité B dans les deux traitements pour un niveau donné de pénalité, $F$, et pour des valeurs de $p$ et de $n$ qui mettent en œuvre un risque identique de détection: $p \approx n / 20$. A l'inverse, un participant peut être considéré comme optimiste (respectivement pessimiste) s'il affecte plus (moins) de jetons à l'activité risquée (option B dans l'expérience) dans le traitement Désignation que dans le traitement Urne individuelle. Le risque étant maintenu constant, cet écart correspond en effet à une déformation de la probabilité perçue entre les deux traitements. L'hypothèse de travail au regard de laquelle les données expérimentales sont analysées résume ces éléments.

\footnotetext{
${ }^{2}$ Pour Experimental Currency Unit, l’unité monétaire utilisée dans les expériences.

${ }^{3}$ Le protocole de l'expérience a été réalisé sous le logiciel REGATE (Zeiliger, 2000).
} 
Hypothèse: En l'absence de déformation en raison du biais d'optimisme, l'investissement dans l'activité risquée est, toutes choses égales par ailleurs, indépendant des modalités du contrôle.

Les statistiques descriptives de l'investissement dans l'activité risquée (Tableau l) suggèrent une forte remise en cause de cette hypothèse. Un changement de décision est en effet observé dans plus de $53 \%$ des cas (soit 1023 décisions). Ces changements sont en moyenne positifs, allant dans le sens d'une sous-estimation de la probabilité (i.e. optimisme) dans le traitement Désignation. Cette tendance moyenne s'accompagne cependant d'une très forte dispersion, suggérant une importante idiosyncrasie de la sensibilité de l'optimisme aux modalités du contrôle.

Avant d'évaluer la significativité statistique de ces observations, le Tableau 2 présente, à titre de vérification, une analyse des déterminants des décisions d'investissement dans l'activité risquée dans chacun des deux traitements. Il convient de rappeler à cet égard que le nombre de jetons investis dans l'activité risquée est borné inférieurement (à 0) et supérieurement (à 20) dans le cadre de l'expérience. La spécification utilisée tient compte de cette censure sous forme d'un modèle Tobit (type 1) à deux bornes. En outre, 54 décisions successives sont observées pour chaque individu. Un effet individuel aléatoire est donc ajouté au modèle afin de prendre en compte cette dimension de panel.

Tableau 1. Comportement d'investissement dans l'activité risquée

1a. Amende faible $(F=10)$. Amende élevée $(F=14)$
\begin{tabular}{r|c|c|r|rc|r|r|r|} 
Quantités investies $(Q)$ & \multicolumn{2}{c}{ Taux de variation } & \multicolumn{2}{c}{ Quantités investies $(Q)$} & \multicolumn{2}{|c}{ Taux de variation } \\
Prob. & Urne & Désignation & Moy. & Ec. Type & Urne & Désignation & Moy. & Ec. Type \\
\hline \hline 0.05 & 12.47 & 13.77 & 26.79 & 128.33 & 11.98 & 12.77 & 18.79 & 101.81 \\
0.10 & 11.25 & 12.24 & 32.00 & 142.63 & 8.25 & 10.92 & 116.86 & 285.9 \\
0.15 & 9.45 & 9.41 & 9.54 & 70.38 & 6.74 & 8.01 & 50.21 & 170.44 \\
0.20 & 7.05 & 7.38 & 36.94 & 133.42 & 4.9 & 5.92 & 40.33 & 164.1 \\
0.25 & 5.61 & 6.34 & 12.16 & 68.31 & 3.86 & 5.34 & 46.27 & 128.19 \\
0.30 & 4.64 & 4.76 & 31.85 & 161.14 & 3.26 & 3.4 & 25.3 & 169.88 \\
0.35 & 4.18 & 3.29 & 5.54 & 112.32 & 2.4 & 2.38 & 36.6 & 131.28 \\
0.40 & 2.63 & 2.75 & 54.22 & 224.61 & 1.07 & 1.95 & 101.62 & 182.76 \\
0.45 & 1.71 & 1.94 & 1.33 & 93.63 & 0.86 & 0.86 & 51.31 & 191.62 \\
0.50 & 1.44 & 2.36 & 94.46 & 249.41 & 1.01 & 0.68 & 8.76 & 109.43 \\
0.55 & 1.22 & 1.60 & 30.20 & 134.41 & 0.67 & 1.05 & 158.94 & 299.52 \\
\hline \hline Total & 5.61 & 5.99 & 28.29 & 139.99 & 4.09 & 4.84 & 54.18 & 183.77
\end{tabular}

Note : La partie gauche décrit les comportements lorsque l'amende est élevée, la partie droite lorsqu'elle est faible. Dans chaque tableau, pour un niveau de risque donné ( $p \approx n / 20$, première colonne), les colonnes centrales présentent le nombre de jetons investis en moyenne dans l'activité risquée (option B dans l'expérience) dans chacun des traitements Urne individuelle et Désignation. Les deux dernières colonnes présentent la moyenne (première colonne) et l'écart-type (deuxième colonne) du taux de variation en \% de l'investissement entre les deux traitements, i.e. $\left(\mathrm{Q}_{\text {désignation }}-\mathrm{Q}_{\text {urne }}\right) / \mathrm{Q}_{\text {urne. }}$.

Tableau 2. Déterminants de l'investissement dans l'activité risquée

2a. «Urne individuelle »

\begin{tabular}{l|r|r|c}
\multicolumn{1}{c|}{ Quantités $(Q)$} & \multicolumn{1}{c|}{ Coef. } & \multicolumn{1}{c|}{$\mathrm{t}$} & $\mathrm{p}$ \\
\hline Constante & 49.28 & 7.39 & 0.000 \\
Amende $(F)$ & -0.83 & -9.22 & 0.000 \\
Aversion au risque & -5.93 & -10.48 & 0.000 \\
Probabilité $(p=n / 20)$ & -46.85 & -35.50 & 0.000 \\
Autres caractéristiques & \multicolumn{3}{|c}{ oui } \\
\hline \hline$\sigma_{u}$ & 6.92 & 12.44 & 0.000 \\
$\sigma_{\varepsilon}$ & 6.22 & 38.04 & 0.000 \\
$\rho$ & 0.55 & - & - \\
\hline \hline Log-vraisemblance & -3238.62 \\
Wald chi2(23) & 1373.10 \\
Prob $>$ chi2 & 0.00 \\
\hline \hline
\end{tabular}

2b. «Désignation»

\begin{tabular}{l|r|r|c}
\multicolumn{1}{c|}{ Quantités $(Q)$} & \multicolumn{1}{c|}{ Coef. } & \multicolumn{1}{c|}{$\mathrm{t}$} & $\mathrm{p}$ \\
\hline Constante & 51.49 & 7.34 & 0.000 \\
Amende $(F)$ & -0.59 & -7.17 & 0.000 \\
Aversion au risque & -4.47 & -7.92 & 0.000 \\
Probabilité $(p=n / 20)$ & -46.91 & -39.45 & 0.000 \\
Autres caractéristiques & \multicolumn{3}{|c}{ oui } \\
\hline \hline$\sigma_{\mathrm{u}}$ & 7.46 & 12.52 & 0.000 \\
$\sigma_{\varepsilon}$ & 5.82 & 39.08 & 0.000 \\
$\rho$ & 0.62 & - & - \\
\hline \hline Log-vraisemblance & \multicolumn{3}{|c}{-3323.43} \\
Wald chi2(23) & 1633.16 \\
Prob > chi2 & 0.00 \\
\hline \hline
\end{tabular}

Note : Tobit à effets individuels aléatoires. Variable endogène : nombre de jetons investis dans l’activité risquée (option B) dans le traitement Désignation (Tableau 2a) et dans le traitement Urne individuelle (Tableau 2b). Les caractéristiques individuelles dont les coefficients ne sont pas reportés sont : l’âge, le sexe, le statut matrimonial, le nombre d'enfants, le statut d'activité et le diplôme de l'individu, le diplôme de sa mère ainsi que celui de son père. 
Dans l'ensemble, les résultats sont à la fois conformes à l'intuition et concordants avec les résultats attendus. Quel que soit le traitement, l'investissement dans l'activité risquée apparaît en effet décroissant de la probabilité de contrôle, du niveau de la pénalité et du degré d'aversion au risque.

Tableau 3. Effet du traitement « Désignation » sur l'investissement dans l'activité risquée

\begin{tabular}{l|r|r|c}
\multicolumn{1}{c|}{ Quantités $(Q)$} & \multicolumn{1}{c|}{ Coef. } & \multicolumn{1}{c|}{$\mathrm{t}$} & $\mathrm{p}$ \\
\hline Constante & 47.68 & 9.01 & 0.000 \\
Traitement & 1.28 & 4.92 & 0.000 \\
Amende $(F)$ & -0.72 & -11.02 & 0.000 \\
Aversion au risque & -5.11 & -12.36 & 0.000 \\
Probabilité $(p=n / 20)$ & -47.60 & -50.42 & 0.000 \\
Autres caractéristiques & \multicolumn{3}{|c}{ oui } \\
\hline \hline$\sigma_{\mathrm{u}}$ & 7.66 & 12.83 & 0.000 \\
$\sigma_{\varepsilon}$ & 6.48 & 54.81 & 0.000 \\
$\rho$ & 0.58 & - & - \\
\hline \hline \hline Log-vraisemblance & -6555.94 \\
Wald chi2(23) & 2077.97 \\
Prob > chi2 & 0.00 \\
\hline \hline
\end{tabular}

Note : Tobit à effets individuels aléatoires. Variable endogène : nombre de jetons investis dans l'activité risquée (option B) tous traitements confondus. La variable Effet de traitement correspond à une variable indicatrice du traitement (= 1 pour le traitement Désignation). Les caractéristiques individuelles dont les coefficients ne sont pas reportés sont: l’âge, le sexe, le statut matrimonial, le nombre d'enfants, le statut d'activité et le diplôme de l'individu, le diplôme de sa mère ainsi que celui de son père.

Si les comportements d'investissement dans chaque traitement sont globalement conformes aux résultats attendus, les variations entre traitements indiquent quant à elles un fort effet de la politique de communication. Le Tableau 3 présente les résultats d'une régression (modèle Tobit à effets individuels aléatoires) où les deux traitements sont regroupés. L’effet des propriétés de la loterie (amende, probabilité) et des caractéristiques individuelles (aversion au risque) va dans le même sens que dans les régressions précédentes, où les coefficients dépendent du traitement considéré. Cette spécification permet cependant d'estimer l'effet de traitement, c'est-àdire l'influence toutes choses égales par ailleurs de la politique de communication sur les décisions d'investissement. La désignation d'un nombre fixe de participants plutôt que l'annonce du niveau de la probabilité de contrôle augmente de 1.27 jetons en moyenne le niveau d'investissement dans l'activité risquée. Cet effet -- significatif à plus de 1\% -- confirme sans ambigüité qu'une politique de communication fondée sur la désignation accroît l'optimisme des fraudeurs potentiels.

\section{CONCLUSION}

Prenant acte des développements récents des recherches en psychologie, cet article propose une analyse expérimentale de l'effet de l'optimisme sur les décisions de se livrer à une activité illégale. Il ressort nettement que d'autres ressorts psychologiques que l'aversion au risque affectent les décisions de fraude. Adopter un point de vue holistique (nombre de membres du groupe désignés aléatoirement à subir un contrôle) tend à encourager la fraude en comparaison avec un point de vue idiosyncrasique (probabilité attachée à la loterie individuelle).

Ces résultats élargissent le champ des leviers d’une politique active de gestion des activités illégales, souvent restreint à la fréquence du contrôle et aux pénalités en cas de détection. La perception du contrôle, à travers le degré d'optimisme induit par la politique de communication, se révèle en effet déterminante dans les décisions. L'usage qui peut en être fait dépend des objectifs assignés au contrôle. Les autorités peuvent en effet souhaiter encourager (en favorisant l'optimisme) ou, au contraire, décourager ces comportements selon que le contrôle est conçu comme : (i) un instrument de filtrage permettant de détecter les agents économiques enclin à se livrer à des activités illégales; ou (ii) un élément du coût des activités illégales, auquel réagissent des agents économiques opportunistes.

\footnotetext{
4 Parmi les variables dont les coefficients ne sont pas présentés dans le Tableau 2, il convient de signaler que le niveau d’éducation, le nombre d'enfants à charge et le niveau d'éducation de la mère contribuent à réduire l'investissement dans l'activité risquée ; tandis que l'âge, le niveau d'éducation du père et le fait d’être marié tendent à augmenter l'investissement dans l'activité risquée.
} 
Chacun de ces objectifs repose sur des hypothèses de comportement particulières, dont la pertinence reste variable selon le type d'activité illégale considérée. Le premier paraît ainsi approprié à une situation dans laquelle seules certaines catégories de population sont susceptibles de frauder, et ce de façon peu élastique aux instruments de répression ; le second décrivant quant à lui une situation de fraude conçue comme un problème d'incitation. Les résultats présentés permettent de mettre la politique de communication au service de l'un ou l'autre de ces objectifs, à travers un accroissement ou une réduction de l'optimisme sous-jacent aux décisions.

\section{RÉFÉRENCES BIBLIOGRAPHIQUES}

ALICKE M. D. [1985]: "Global self-evaluation as determined by the desirability and controllability of trait adjectives", Journal of Personality and Social Psychology, Vol. 49, No. 6, p.1621-1630.

BECKER G. S. [1968]: "Crime and Punishment: An Economic Approach", Journal of Political Economy, Vol. 76, No. 2, p.169-217.

BENABOU R., TIROLE J. [2002]: "Self-confidence and personal motivation", Quarterly Journal of Economics, Vol. 117, No. 3, p.871.

BIAIS B., HILTON D., MAZURIER K., POUGET S. [2005]: "Judgemental Overconfidence, Self-Monitoring, and Trading Performance in an Experimental Financial Market", Review of Economic Studies, Vol. 72, No. 251, p.287-312.

CAMERER C., LOVALLO D. [1999]: "Overconfidence and Excess Entry: An Experimental Approach", American Economic Review, Vol. 89, No. 1, p.306-318.

FANG H., MOSCARINI G. [2005]: "Morale hazard", Journal of Monetary Economics, Vol. 52, No. 4, p.749777.

FISCHHOFF B., SLOVIC P., LICHTENSTEIN S. [1977]: "Knowing with certainty: The appropriateness of extreme confidence", Journal of Experimental Psychology: Human Perception and Performance, Vol. 3, No. 4, p.552-564.

GERVAIS S., ODEAN T. [2001]: "Learning to be Overconfident", Review of Financial Studies, Vol. 14, No. 1, p.1-27.

GRIFFIN D., TVERSKY A. [1992]: "The weighing of evidence and the determinants of confidence", Cognitive Psychology, Vol. 24, No. 3, p.411-435.

HOELZL E., RUSTICHINI A. [2005]: "Overconfident: Do You Put Your Money On It?" Economic Journal, Vol. 115, No. 503, p.305-318.

HOLT C. A., LAURY S. K. [2005]: "Risk Aversion and Incentive Effects: New Data without Order Effects", American Economic Review, Vol. 95, No. 3, p.902-905.

INTERNAL REVENUE SERVICE, [1996]: "Federal Tax Compliance Research: Individual Income Tax Gap Estimates for 1985, 1988, and 1992", Research Division Publication, Vol. 1415-E.

WEINSTEIN N. D. [1980]: "Unrealistic optimism about future life events", Journal of Personality and Social Psychology, Vol. 39, No. 5, p.806-820.

ZEILIGER R. [2000]: "A presentation of Regate, Software for Experimental Economics", http://www.gate.cnrs.fr/ zeiliger/regate/regate.htm. 